\title{
Intelligent support tools for strategic decision-making on Smart Grid development
}

\author{
Liudmila Massel $^{1}$, Aleksey Massel $^{1}$ \\ ${ }^{1}$ Melentiev Energy System Institute of SB RAS, Lermontov str., 130, Irkutsk, Russia
}

\begin{abstract}
The article discusses the concepts of Smart Grid and the digital power industry being developed in Russia. The main information technologies offered for their implementation are analyzed. It is proposed to include in this list intelligent technologies to support strategic energy development decisions,. Three generations of such tools developed in a team headed by the authors are considered. The proposed Intellectual tools are based on the concepts of situational management and semantic modeling. It is noted that the third version seems to be the most perspective. Examples of semantic models and schemes of the proposed intelligent tools are given.
\end{abstract}

\section{Introduction}

At present, the world to promote actively the conception, called Smart Grid (intelligent energy systems). Smart Grid is electric power systems with the use of new technologies such as in the power equipment, as well as in the field of information support, aimed at improving the quality of technical and economic parameters [1-2].

Now, within the framework of the Digital Economy concept in Russia, the project "Digital Energy" was created [3-4]. Its authors note:

- In the field of digital technologies in power engineering in Russia there are a significant results.

- Digital energy development requires the development and consolidation of these results in the form of industry digital platforms, that is, a comprehensive digital transformation of the energy industry

- The organizational and financial support for such digital transformation, similar to other industries, should be the main task of the federal project "Digital Energy".

At last time Scientific and Expert Council under the working group of the Council of Federation on monitoring the implementation of legislation in the field of energy, energy conservation and energy efficiency was created. One of the authors was invited to be a member of Section «Digital Technologies in Energy and Energy Saving» in this Council. One of the tasks of this section: the development of the concept of "Digital Energy". The following are the suggestions and results of the authors that can be used in the implementation of this project.

\section{The main recommended information technologies for digital energy:}

- Industrial Internet (Internet of things in the industry) for telemetry of power system parameters.

- Technologies for analyzing large data (BigData) in order to predict the behavior of the power system.
- BIM (Building Information Model) - technologies for energy facilities (power plants, substations, production and processing facilities, etc.).

- GIS-technologies for energy facilities associated with transportation of fuel and energy resources (power lines, pipelines, etc.).

- Technology of remote sensing of the Earth for observation of natural and technogenic factors.

- Satellite navigation systems for monitoring discrete transport in conjunction with GIS-technologies [3].

Section "Digital Technologies in Energy and Energy Saving" proposed to supplement this list with the following technologies:

- Neurotechnologies and artificial intelligence - for System of automatic regime control and maintenance of stability of power systems (Neural Grid)

- Distributed Registry Systems - for Blocheinenvironment of energy trade, equipment services (Transactive Energy)

- Wireless technology - for Wireless communication between digital power equipment

- Technologies of virtual and augmented realities for Digital Twins of power systems

We proposed to include in this list intelligent technologies to support the adoption of strategic decisions on the development of intelligent and digital energy.

In addition, the concept of digital economy and digital energy does not include issues of cybersecurity. We decide that cyber danger is a strategic threat to energy security, and this threat will be intensified in the transition to intelligent and digital energy.

We understand cybersecurity according to international standard ISO 27032: 2012. Cybersecurity is based on five components (Information Security, Application Security, Network Security, Internet Security and Critical Information Infrastructure Protection), but is not a synonym of one of them.

The following substantiates the need for the development of intelligent DSS [5]. 
In the intelligent and digital energy, two interconnected areas are singled out - the technological infrastructure and the information and communication infrastructure. The success of creating intelligent energy systems largely depends on the successful application of modern information technology. In turn, talk about the application of the latter makes sense when there is a developed modern technological infrastructure.

Decisions on the development of technological infrastructure, of course, belong to the class of strategic decisions. To justify and support the adoption of such decisions, it is advisable to attract intelligent information technologies. This, in particular, the technology of situation management, semantic modeling and knowledge management, which are developed under the guidance of the authors.

\section{Situational management}

Russian scientist D.A. Pospelov was a founder of the direction «Situational management» [6]. $\mathrm{He}$ introduced following definitions:

- The current situation $(Q j)$ - the set of all information about the structure of the object of control and its operation at any given time.

- Complete situation ( $\mathrm{Si}$ ) - the collection consisting of the current situation, knowledge of the state management system at this moment and on the knowledge about management technology.

- Logical and transformation rules (LTR) - the conversion rules of the current situation $Q j$ in the current situation $Q l$ as result of control action $U k$

- Classification procedures - procedures that would classify the overall situations, so that one could form as many classes as different single-step solutions is at the disposal of the control system.

- Extrapolation procedures - procedures solve the choice problem of a number of possible solutions out for the full situation.

Taking into account the definitions given above, the general scheme of situational management, according to the D.A. Pospelov includes blocks: Analyzer, Classifier, Correlator, Extrapolator and Block of random selection. The comparison of these blocks with author's tools of semantic modeling will be given later.

We use modern treatment of situational management, the essence of which lies in the choice of management solutions tailored to the situation of a certain set of admissible (typical, standard) control actions. Under the current situation it's understood the totality of the current state of the object (the state vector $\mathrm{X}$ ) and its external environment (vector of perturbations F).

Then $\mathrm{C}=\langle\mathrm{X}, \mathrm{F}\rangle$. Also the concept of complete situation is introduced: $\mathrm{S}=\langle\mathrm{C}, \mathrm{G}\rangle$, where $\mathrm{C}-$ the current situation, $\mathrm{G}$ - management goal. In turn, the control target $\mathrm{G}$ can be represented as a target situation
Gg, which should be given the available current situation. Then $\mathrm{S}=\langle\mathrm{C}, \mathrm{Gg}\rangle$.

In other words, when we use the situational management, problem of choice of control actions is reduced to an adequate assessment of the object state and the environment (which is complicated by the presence of uncertainty factors), to the attribution of the appropriate current situation to one of the standard classes and to selection of such control (from a particular set of alternatives), which leads to the achievement of the control target (the target situation).

We use the term Contingency management instead of Situational management. What is the reason? Since the results obtained by the authors, are used in a field related to energy security (ES), situational management in this context is regarded as a contingency management (management in extreme situations in the energy sector) [7].

Under extreme situations (ExS) in the energy sector are understood as emergency and critical situations, the determination of which is based on the assessment of the status of systems or objects on the scale of "normal", "pre-_crisis" - a critical situation, "crisis" - an emergency.

On this basis, under the critical situations defined as a situation where there are threats to the smooth functioning of the technical facilities and life support and / or threat to the life or health of individuals and social (professional) groups. These threats can be eliminated by the adoption of appropriate preventive and operational measures, which will not allow the critical situation to develop into an emergency.

Currently, attention is paid mainly support decisionmaking and management in emergency situations where the implementation of operational and liquidation activities, it is directed, including the activities of units of Ministry of Emergency Situations.

The authors give preferential attention to modeling and analysis of critical situations, allows you to create a list of preventive and operational measures to prevent possible emergencies.

Fig. 1 is shown interpretation of contingency management by the example of Fuel and Energy Complex (FEC) research.

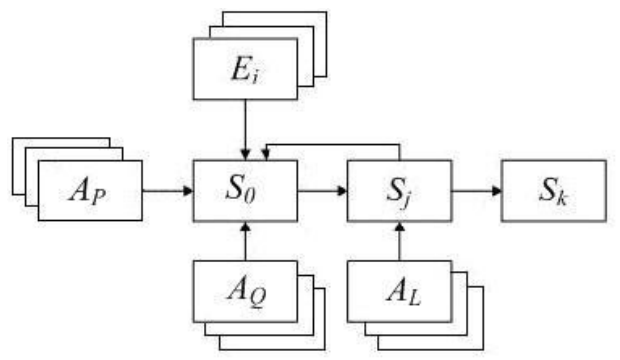

Fig. 1. Interpretation of contingency management by the example of energy research 
Here So is the initial state of FEC, can be considered as the current situation $\boldsymbol{C} ; E i$ - scenarios of possible extreme situations in case of ES-threats realization (similar external influences $\boldsymbol{F}) ; A p, A q, A l$ - a set of preventive, operational and liquidation activities to prevent, neutralize or mitigate the effects of an emergency situation (can be seen as a set of related controls $\boldsymbol{U})$ ); $S j$ - the FEC state after the emergency (realization of ES-threats), taking into account a set of implementation liquidation activities; $S k$ - FEC state after liquidation measures (can be seen as analogous to the corresponding target situation $\boldsymbol{G}_{\boldsymbol{g}}$ )).

\section{Semantic modeling}

The semantic modeling is one of the modern trends of artificial intelligence and is associated with the description of the subject area with the help of concepts (basic concepts) and relations between them. The group by leading of authors developed these types of semantic modeling as an ontological, cognitive, event and probabilistic modeling [8].

The first (the ontological) is used to classify domain concepts, the second (cognitive) allows you to identify causal relationships between concepts (factors) and the contribution of factors (positive or negative), for example, in the formation of the threats to energy security. Probabilistic and event simulation in this case are used to model the development of critical and emergency situations resulting from the implementation of energy security threats.
Ontologies have been proposed for declarative knowledge representation and are defined in general terms as the knowledge base of a special form, or as a "specification of a conceptualization" domain $[9,10]$.

This means that in a domain based on the classification of basic terms are basic concepts (conceptualization) and establish links between them (specification).

Then ontology can be represented in graphical form, or is written in a formal language (formal ontology) - a process ontology specifications. The group representing authors, was developed tool OntoMap to support the ontological modeling (can also may be used and other open source tools ontological modeling).

Under cognitive modeling refers to the construction of cognitive models, or in other words, cognitive maps (directed graphs), in which the top of the relevant factors (concept), and the arcs - relations between the factors (positive or negative), depending on the nature of the causal relationship [11].

Mathematical tool for building cognitive models is the theory of graphs. Cognitive modeling in the energy sector was used by auturthes for situational analysis of the ES problem and threat modeling. The group by leading of authors implemented tools to support cognitive modeling - Library CogMap.

On the fig. 2 is shown the example of cognitive map for cold snap threat.

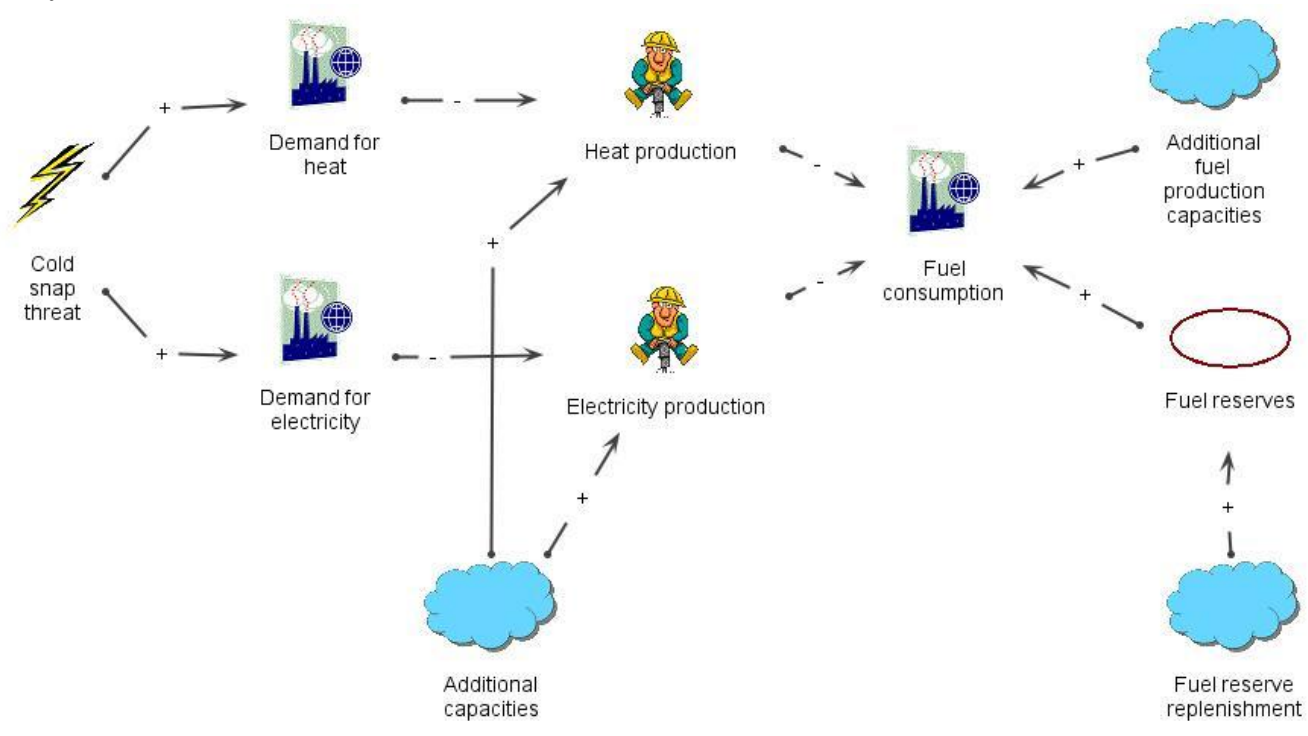

Fig. 2. A cognitive map of a natural threat (cold snap)

By event simulation is meant to build behavioral models, and as objects of modeling can be seen as people and technical facilities. The essence of the event simulation method consists in tracing the sequence of events on the model in the same order in which they would occur in a real system (fig.3). 
Asked model consistent implementation of events a chain of events - describe the scenario of system response to the occurrence of a triggering event, standing at the beginning of the chain.

As a tool for event simulation apparatus used Joinernetworks (JN) - a form of algebraic networks, which can be regarded as an extension of Petri nets, focused on the construction of behavioral patterns. The theory $\mathrm{JN}$ is the description logic of interaction asynchronous processes as a set of launchers and flag functions, consisting of Boolean functions [12]. JN special feature is that they provide both a graphical representation and a description in the form of logical formulas that can automate the process (fig. 4). Library EventMap is implemented as support tools for event modeling.

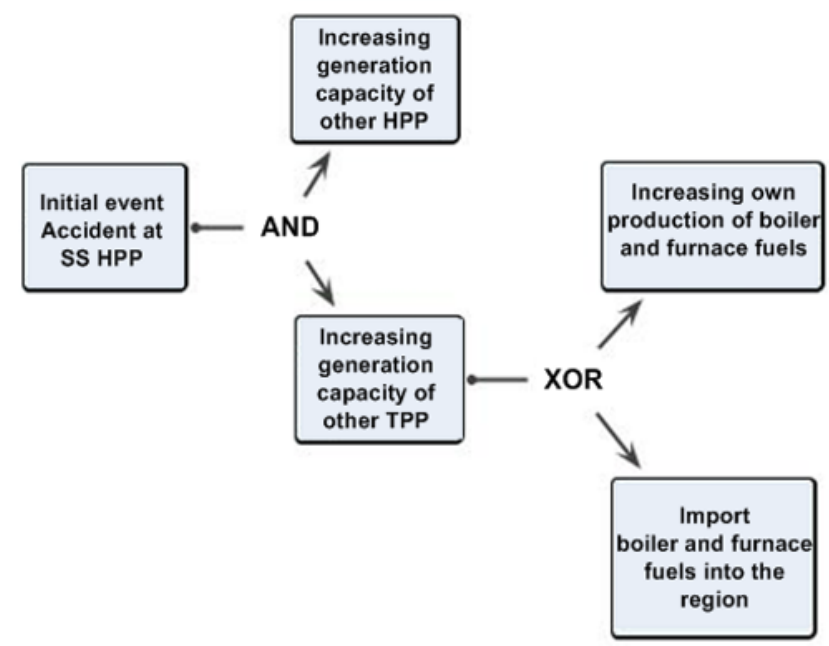

Fig. 3. Event map of development and consequences of accident at Sayan-Shushenskaya hydro-power plant

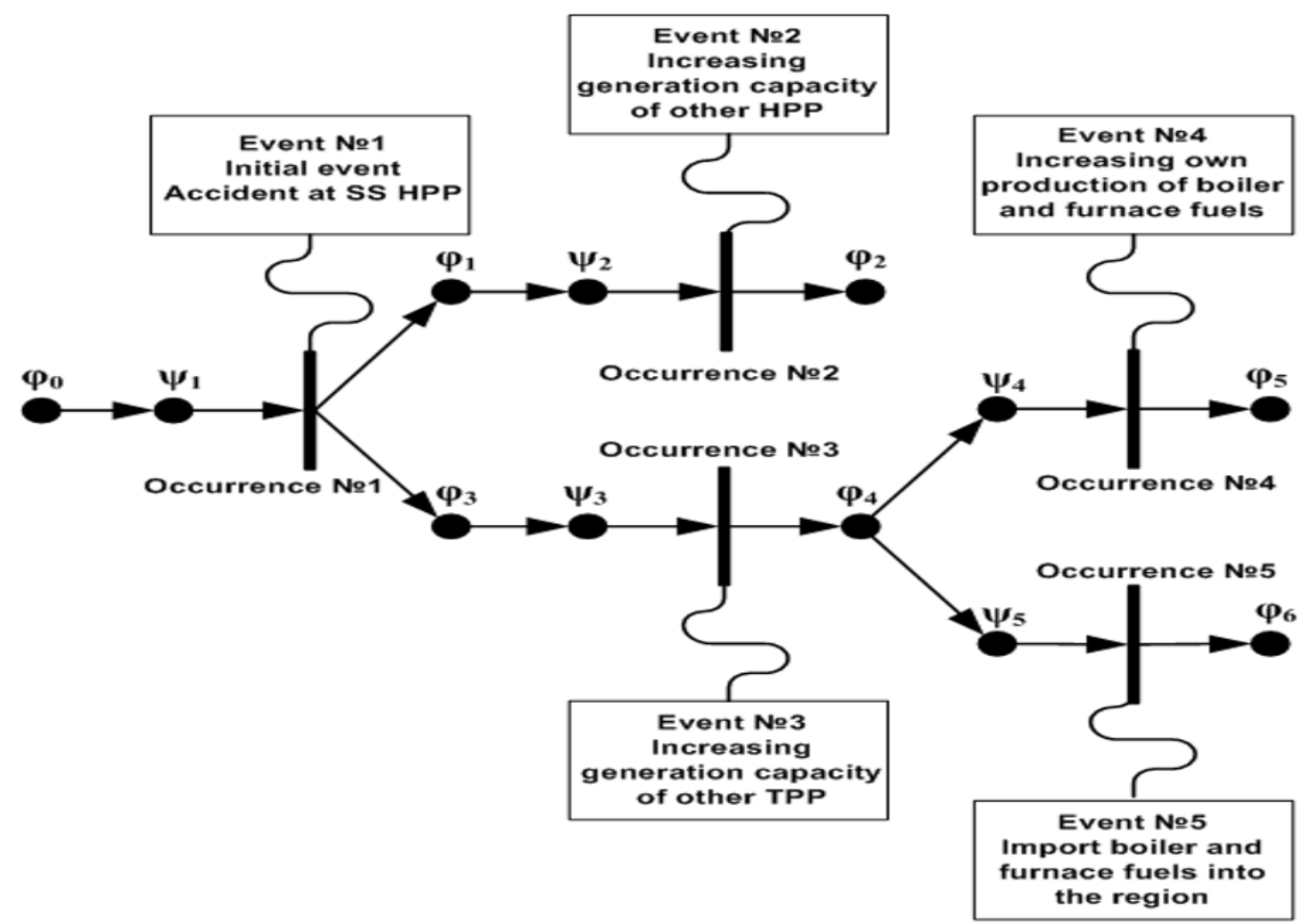

Fig. 4. Example of Joiner-net for event map at the fig. 3.

The comments to fig. 4 :

\begin{tabular}{|c|c|}
\hline Starting functions: & Denoting (flag) functions: \\
$\psi_{1}(t+1)=\varphi_{0}(t) \cdot \overline{\varphi_{1}(t)} \cdot \overline{\varphi_{2}(t)} ;$ & $\varphi_{0}(t+1):=0 ; \varphi_{1}(t+1):=1 ; \varphi_{2}(t+1):=1 ;$ \\
$\psi_{2}(t+1)=\varphi_{1}(t) \cdot \overline{\varphi_{4}(t)} \cdot \overline{\varphi_{5}(t) ;}$ & $\varphi_{1}(t+1):=0 ;\left(\varphi_{4}(t+1):=1\right) \vee\left(\varphi_{5}(t+1):=1\right)$ \\
$\psi_{3}(t+1)=\varphi_{2}(t) \cdot \overline{\varphi_{3}(t)} ;$ & $\varphi_{2}(t+1):=0 ; \varphi_{3}(t+1):=1 ;$ \\
$\psi_{4}(t+1)=\varphi_{4}(t) \cdot \overline{\varphi_{6}(t)} ;$ & $\varphi_{4}(t+1):=0 ; \varphi_{6}(t+1):=1 ;$ \\
$\psi_{5}(t+1)=\varphi_{5}(t) \cdot \overline{\varphi_{7}(t)} ;$ & $\varphi_{5}(t+1):=0 ; \varphi_{7}(t+1):=1 ;$ \\
\hline
\end{tabular}


In recent years, the area of semantic modeling was extended of using of probabilictic modeling on basis of Bayesian belief networks for assessing the risks of emergency situations, which is implemented to support the library BayNet.

All semantic models are usually available in graphical form, although each type of semantic model has a mathematical or formal apparatus to describe them: a special formal language for describing ontologies (XML, RDF, OWL, and others.), Graph theory for cognitive models, the theory of Bayesian belief networks for probabilistic and theory of networks Joiner-event models).

Semantic modeling tools were integrated into the framework of Intelligent IT- environment (fig. 5) [8]

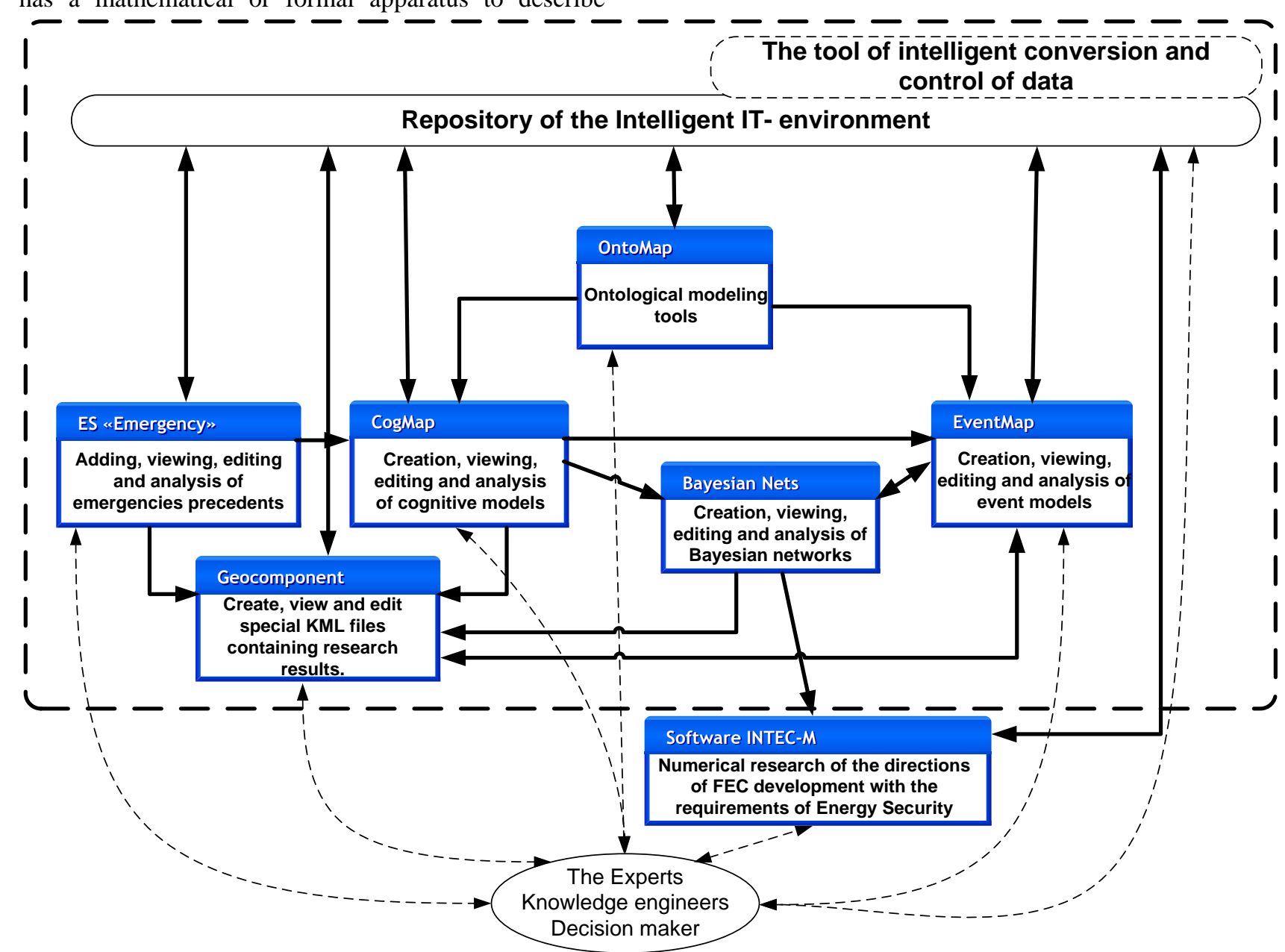

Fig. 5. Intelligent IT- environment for semantic modeling

\section{Intelligent tools for decision support}

At this scheme (fig. 5) OntoMap, CogMap, EventMap and Bayesian Nets are copyright tools for semantic modeling. Geo-component is tool for 3Dgeovisualization, Expert System contains the descriptions of emergency precedents in energy sector. The Repository provides storage of descriptions of all components of this scheme. These components are used for qualitative analysis of situations and the generation of recommended solutions. To substantiate the recommended solutions, it is proposed to use Software INTEC-M at the following quantitative level. The tool of intelligent conversion and control of data provides integration of tools for semantic and mathematical modeling.
The authors compared the components of the general scheme of situational management (by D.A. Pospelov) and the copyright tools of semantic modeling. It is proposed to use the Contingency management language and the Expert System for the implementation of the Analyzer block. The functions of the Classifier block can be performed by the expert system and tools OntoMap and CogMap. To implement the Extrapolator block, it is proposed to use the tools EventMap and ByNet, as well as CogMap to implement the functions of the Correlator block.

On the base of this comparison the architecture of the Situation polygon as an intelligent system for support of strategic decisions in the energy sector was proposed (fig. 6) [13, 14].

This architecture includes the basis components of Intelligent IT- environment for semantic modeling, Knowledge base for descriptions storing of situations, 
emergency scenarios and control actions. Expert system Advise is used for formation and analysis of these descriptions, and for generation of recommended solutions. Contingency Management Language (CML) is proposed to use for several purposes, such as:

1) the language for knowledge description and knowledge manipulation;

2) the tool for situations classification ("norm", critical situations, emergency situations);

3 ) the means of expert system initiation to establish a correspondence between situations and control actions;

4) means for calling the appropriate tools of semantic modeling and mapping modules (to transit from one model type to another);

5) means of recourse to geocomponent for $3 \mathrm{D}$ geovizualization of modeling results.

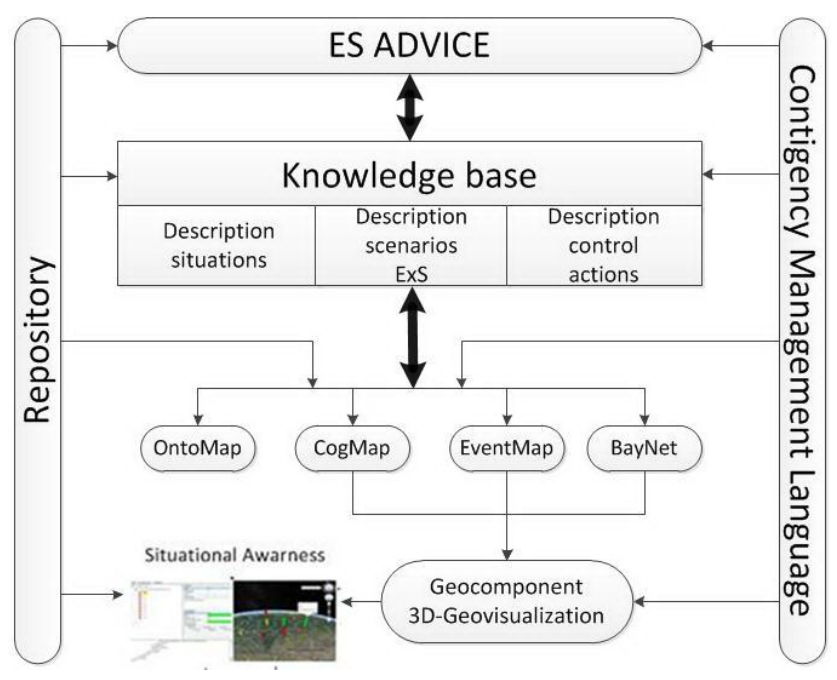

Fig 6. The architecture of the Situation polygon

"Immersion" of researcher in visual environment for solving of spatial problems with help of Geocomponent for 3D-geovisualisation provides situational awareness, which is certainly due to the activation of the subconscious mind and improves the quality of tasks, involving intuition and experience.

The integration of the intelligent IT-environment and the Situation polygon allowed us to move on to the next generation of Intelligent decision-making support tools in the energy sector.

7 Multi-agent intelligent environment for substantiation of strategic decisions on the energy development.

Now we propose to use the integrated research carried out at the institute to forecast the development of energy, as a scientific basis of for substantiation of strategic decisions on the energy development, at first turn as Smart Grid and Digital Energy. The four levels (stages) of research and tools for their support are defined.

These are next levels:

1) the level of analysis (semantic modeling), supported by Intelligent IT-environment;

2) the level of proposed decisions (collective expert activity), with help of Intelligent system for supporting of collective expert activity;

3) the level of decisions justification (mathematical modeling) supported by software systems;

4) the level of presentation of recommended solutions with help of visual analytics and cognitive graphics.

The architecture of the multi-agent intelligent environment supporting these levels is shown on the fig. 7 .

This architecture includes:

- Software Systems and Data Bases for research of the fuel and energy complex together with Software and Data Bases, for example, for energy security research;

- Data and Knowledge Warehouse;

- Intelligent system for supporting of collective expert activity;

- Intelligent IT-environment for supporting of semantic modeling;

- Software component for visual analytics (GEO-visualization component);

- Portal - Ontological Knowledge Spase.

Knowledge Management Language is used for integration of these components and for call of the required component. The Repository provides storage of descriptions of all components, as well as databases and knowledge bases.

Now we have scientific prototypes of all basis components of this scheme. Their adaptation, integration and approbation is required to solve practical problems in this area.

The principles outlined above are used also in the our International science project «Methods and technologies for assessing the impact of energy on the geoecology of the region», supported by Eurasian Association for the Support of Scientific Research and Russian Fund of Basis Research. The project is implemented jointly with science collectives from Belarus and Armenia. The object of research in Russia is the Baikal natural territory, comparable in size with Belarus and Armenia. We hope that the results of this project will be our contribution to the development of Green Energy. 


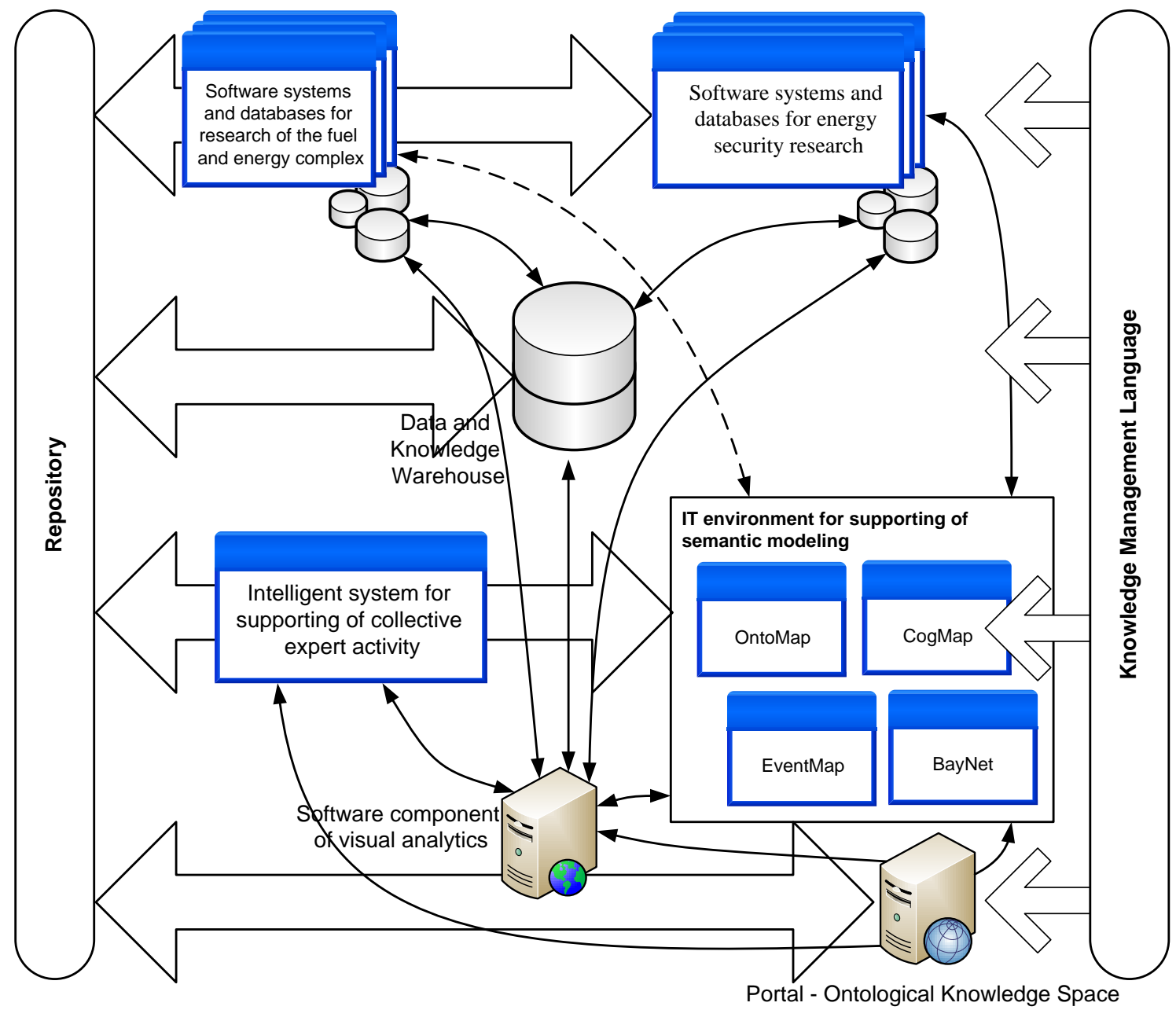

Fig. 7. The architecture of the multi-agent intelligent environment

\section{Conclusion}

The article justifies the need to develop intelligent DSS to support the adoption of strategic decisions in Intelligent (Smart Grid) and Digital energy. We propose to integrate in DSS mathematical and semantic models and visual analytics techniques, including the use of geo-information technologies. The article discusses three versions (generations) of such tools: Intelligent IT- environment for semantic modeling, the Situation polygon for decision support and Multiagent intelligent environment for substantiation of strategic decisions on the energy development. The latest version is based on the integration of modern advances in information technologies. We have scientific prototypes of all basis components of proposed environment. It takes adaptation, integration and approbation of these components to solve practical problems in this area.

\section{Acknowledgement}

The results provided in the article have been acquired under the partial financial support of RFBR grants №16-07-00474, № 18-57-81001，№ 18-0700714.

\section{References}

1. Kobets B.B., Volkova I.O. The innovative development of electric power based on the concept of Smart Grid. M.: IAC Energy. 2010. 208 p. (in Russian).

2. Voropai N.I., Stennikov V.A. Integrated Intelligent Energy Systems // News of the Russian Academy of Sciences. Energy. №1. 2014. Pp. 64-78 (in Russian).

3. The Federal project "Digital Energy" http://minsvyaz.ru/uploaded/files/programma.pdf (access date 08.07.2018)

4. Bushuev V.V. The main provisions of the strategic direction of development of the electric grid complex of Russia. Energetic.- №6, 2018.- Pp.1214 (in Russian).

5. Massel L.V. Problems of the Smart Grid creation in Russia with a view to information and telecommunication technologies and proposed solutions // 14th International Workshop on 
Computer Science and Information Technologies: proceedings. Vol. 1. 2013. Austria- SlovakiaHungaria. Pp. $115-120$.

6. Pospelov D.A. Situational management. Theory and practice. Moscow: Science. 1986. 284 p. (in Russian).

7. Massel L.V., Massel A.G. Contingency management and semantic modeling in energy sector $/ / 15^{\text {th }}$ International Workshop on Computer Science and Information Technologies: proceedings. Vol. 1. 2014. England. Sheffield. Pp. 158-162.

8. Massel L.V., Arshinsky V.L., Massel A.G. Intelligent computing on the basis of cognitive and event modeling and its application in energy security studies // International Journal of Energy Optimization and Engineering. Special issue on: Mathematical and heuristic modeling and optimization of energy systems. № 3(1). 2014. Pp. 83-91.

9. Gruber T. R. Towards principles for the design of ontologies used for knowledge sharing. In $\mathrm{N}$. Guarino and R. Poli, editors, Formal Ontology in Conceptual Analysis and Knowledge Representation. Kluwer Academic Publishers, Deventer, The Netherlands, 1993.

10. Suarez-Figueroa M. C., Gomez-Perez A., Motta E., Gangemi A. (Eds.). Ontology engineering in a networked world. - Springer Science \& Business Media, 2012.

11. Trahtengerc E.A. Computer support of decisionmaking. M.: SINTEG. 1998. 376 p. (in Russian).

12. Stoljarov L.N. The philosophy of event modeling by the example of the emergency scenario in energy sector // International Conference "Information Technologies in science, education, telecommunications and business": Proceedings. Ukraine. Gurzuf // 2010. Pp. 197-200 (in Russian).

13. Massel L.V., Massel A.G. Semiotic approach to development of intelligent systems of contingency management in energy sector/ Proceeding of the Workshop on Computer Science and Information Technologies (CSIT'2015), Rome, Italy.- Vol. 1, 2015.- UFA: USATU.- Pp. 137-144.

14. Massel L.V., Massel A.G. Intelligent system of semiotic type for decision-making support in Russia energy sector based on situational management conception /Proceedings of IV International scientific conference "Information technologies in science, management, social sphere and medicine" (ITSMSSM 2017)) // Advances in Computer Science Research (ACSR). Volume 72, 2017. - Pp. 423-429. https://www.atlantispress.com/ proceedings/itsmssm-17 